CLINICAL STUDY

\title{
Abundant expression of platelet-derived growth factor in spiral arteries in decidua associated with pregnancy-induced hypertension and its relevance to atherosis
}

\author{
Hiroki Morita, Masakazu Mizutori, Kyousuke Takeuchi, Satoru Motoyama and Takeshi Maruo \\ Department of Obstetrics and Gynecology, Kobe University School of Medicine, Kobe, Japan \\ (Correspondence should be addressed to T Maruo, Department of Obstetrics and Gynecology, Kobe University School of Medicine, 7-5-1 Kusunokicho \\ Chuo-ku, Kobe 650-0017, Japan; Fax: +81-78-3826019)
}

\begin{abstract}
Objective: To elucidate the role of platelet-derived growth factor (PDGF) in the pathophysiology of pregnancy-induced hypertension $(\mathrm{PIH})$ in which the spiral arteries of the decidua demonstrate the atherosclerotic change.

Design and Methods: We determined serum levels of PDGF and PDGF expression in the decidua as well as serum levels of $17 \beta$-estradiol (E2) and progesterone (P4) both in normotensive cases and in $\mathrm{PIH}$ cases. Furthermore, we investigated whether sex steroid hormones could interact with PDGF in cultured vascular smooth muscle cells (SMC) by immunohistochemical staining for proliferating cell nuclear antigen.

Results: Serum PDGF levels were higher $(P<0.01)$ but serum E2 levels were lower $(P<0.01)$ in PIH cases compared with normotensive cases. There was no statistically significant difference between serum P4 levels in PIH cases and those in normotensive cases.

Immunohistochemical staining for PDGF in SMC of spiral arteries was more prominent in PIH cases than in normotensive cases. The proliferative potential of cultured SMC was stimulated by PDGF, but inhibited by concomitant treatment with PDGF and E2.

Conclusions: PDGF is suggested to play an important role in the pathophysiology of PIH through its stimulatory effect on vascular SMC proliferation which may elicit the atherosclerotic change in the spiral arteries of the placenta.
\end{abstract}

European Journal of Endocrinology $144271-276$

\section{Introduction}

Platelet-derived growth factor (PDGF) was first identified by Ross et al. while studying wound healing and atherogenesis (1). Since then PDGF has been indicated to have several important roles in cellular proliferation, angiogenesis, tissue injury, and its repair $(2,3)$. In particular, PDGF is widely known to be associated with atherosclerosis by stimulating the proliferation of vascular smooth muscle cells (SMC) and fibroblasts (4).

Pregnancy-induced hypertension (PIH) has been found to show atherosclerotic changes in the spiral arteries of placenta. Zeek and Assali (5) named this change 'acute atherosis'. Acute atherosis is described as an accumulation of fat-containing macrophages in the intima of the maternal decidual vessels, with subsequent fibrinoid necrosis of the media and intimal fibroblastic proliferation (6).

Therefore, it is suggested that PDGF may participate in the pathophysiology of PIH by associating with the atherosclerotic change in the placenta. The purpose of the present study was to elucidate the possible role of PDGF in the pathophysiology of PIH associated with the atherosclerotic change in the spiral arteries in the placenta.

\section{Materials and methods}

\section{Subjects}

The pregnant women participating in this study were recruited in a consecutive manner from the patients at Kobe University Hospital. All women had singleton pregnancies. None of the women had pre-existing hypertensive disorders or renal diseases. After being fully informed about the objectives of the study, each woman gave written informed consent.

We classified the recruited women into three subgroups. The clinical characteristics of each group are indicated in Table 1. One of the subgroups (group A: $n=10)$ consisted of primigravid women whose pregnancies were complicated by severe PIH. The 
Table 1 Clinical characteristics of each group. Data are means \pm S.D.

\begin{tabular}{lccc}
\hline Group & $\boldsymbol{n}$ & Age (years) & Gestational age (week) \\
\hline A Severe PIH & 10 & $28.0 \pm 4.9$ & $31.9 \pm 1.4$ \\
B Preterm delivery & 30 & $27.9 \pm 2.0$ & $31.2 \pm 1.6$ \\
C Normotensive & 30 & $28.0 \pm 3.9$ & $31.7 \pm 1.6$
\end{tabular}

* When blood sample was collected (groups A and C) or at delivery (group B).

clinical manifestations of this group are shown in Table 2. The mean age of this group was $28.0 \pm 4.9$ years. The mean gestational age when blood was collected was $31.9 \pm 1.4$ weeks. In the present study, severe PIH was defined using the criteria of the American College of Obstetricians and Gynecologists (7). All the women who developed severe PIH had been treated with only diet and rest until they delivered or underwent Cesarean section. The blood samples from the PIH women were obtained within $48 \mathrm{~h}$ prior to delivery or Cesarean section. Another subgroup (group B: $n=30$ ) consisted of primigravid women whose pregnancies were complicated by preterm delivery. This subgroup was used as a control group for immunohistochemical study of the decidua. The mean age of this group was $27.9 \pm 2.0$ years. The mean gestational age when delivered was $31.2 \pm 1.6$ weeks, which was similar to that of group A. The patients of this group were recruited under the following conditions: (i) spontaneous delivery at 34 weeks' gestation or earlier; (ii) no evidence of chorioamnionitis. There was no case associated with premature rupture of the membranes in this group. The criteria used to define the absence of chorioamnionitis were: (a) below the cut off levels $(1.5 \mu \mathrm{g} / \mathrm{ml})$ of granulocyte elastase of cervical mucus and (b) below the cut off levels $(0.3 \mathrm{mg} / \mathrm{dl})$ of serum C-reactive protein levels. All the placentae were examined histopathologically after delivery and there were no findings of chorioamnionitis.

For blood sampling, normotensive primigravid women were also recruited as a control group (group C: $n=30$ ) for comparative analysis of serum levels of
PDGF, 17ß-estradiol (E2), and progesterone (P4). The mean gestational age of this group when blood samples were taken was $31.7 \pm 1.6$ weeks, which was similar to that of group A.

\section{Materials}

Phenol red-free Dulbecco's modified Eagle's medium (DMEM) (8, 9)and antibiotic solution $\left(1 \times 10^{5} \mathrm{U} / \mathrm{l}\right.$ penicillin and $50 \mathrm{mg} / \mathrm{l}$ streptomycin) were purchased from Life Technologies (Grand Island, NY, USA). Fetal bovine serum (FBS), E2, and P4 were obtained from Sigma Chemical Co. (St Louis, MO, USA). Monoclonal antibody to the human PDGF-B chain was obtained from Mochida Pharmaceutical Co. (Tokyo, Japan) (10). Monoclonal antibody to human proliferating cell nuclear antigen (PCNA) was purchased from Calbiochem-Novabiochem International (Uniondale, NY, USA). Human umbilical artery SMC were obtained from Technoclone (Wien, Austria). PDGF-BB was purchased from Genzyme (Cambridge, MA, USA).

\section{Blood sampling}

Venous blood samples were taken from each individual of groups $\mathrm{A}$ and $\mathrm{C}$ into pre-chilled blood collection tubes (Sherwood Medical, Ballymoney, UK) as follows: a $1 \times 10 \mathrm{ml}$ sample into a lithium heparin anticoagulant tube, and a $1 \times 10 \mathrm{ml}$ sample into an ethylenediaminetetra-acetic acid (EDTA) anticoagulant tube. All samples were kept at $4{ }^{\circ} \mathrm{C}$ until separated. Plasma was separated from the heparinized sample within two hours of collection to minimize any storage effects. All plasma samples were stored at $-20{ }^{\circ} \mathrm{C}$ until they were analyzed. EDTA samples were used for E2 and P4 analysis.

\section{Serum PDGF, E2 and $P 4$ assays}

Serum PDGF levels were assayed by standard enzyme immunoassay procedures. The standard curve was generated with a recombinant human PDGF (Genzyme).

Table 2 Clinical characteristics of pregnant women with severe PIH.

\begin{tabular}{|c|c|c|c|c|c|c|}
\hline Case no. & Age (year) & Gravidity and parity & Ante pretibial edema & Blood pressure $(\mathrm{mmHg})$ & Proteinuria (mg/dl) & Outcome \\
\hline 1 & 29 & G3P1 & - & $130 \sim 190 / 70 \sim 110$ & - & CS at $29 w$ \\
\hline 2 & 26 & G2P1 & - & $135 \sim 200 / 80 \sim 110$ & 65 & CS at $32 w$ \\
\hline 3 & 20 & G1P1 & - & $120 \sim 180 / 70 \sim 95$ & - & $\mathrm{CS}$ at $31 \mathrm{w}$ \\
\hline 4 & 27 & G3P1 & + & $140 \sim 200 / 80 \sim 105$ & - & CS at $33 w$ \\
\hline 5 & 36 & G2P1 & - & $125 \sim 185 / 80 \sim 100$ & 65 & VD at $32 w$ \\
\hline 6 & 34 & G1P1 & - & $140 \sim 205 / 90 \sim 115$ & - & $\mathrm{CS}$ at $32 \mathrm{w}$ \\
\hline 7 & 22 & G1P1 & - & $130 \sim 185 / 75 \sim 95$ & - & $\mathrm{CS}$ at $31 \mathrm{w}$ \\
\hline 8 & 31 & G2P1 & - & $145 \sim 205 / 85 \sim 110$ & - & CS at $34 w$ \\
\hline 9 & 26 & G1P1 & - & $140 \sim 210 / 85 \sim 115$ & - & CS at $33 w$ \\
\hline 10 & 29 & G3P1 & + & $125 \sim 180 / 65 \sim 95$ & - & VD at $32 w$ \\
\hline
\end{tabular}

G, gravida; P, para; CS, Cesarean section; VD, vaginal delivery; W, weeks. 
Ninety-six-well plates were coated with monoclonal antibody to the human PDGF-B chain, and the bound PDGF was detected by a goat antibody conjugated with horseradish peroxidase. The sensitivity of this assay was $1.0 \mathrm{ng} / \mathrm{ml}$.

Serum E2 and P4 levels in EDTA samples in both groups $\mathrm{A}$ and $\mathrm{C}$ were measured by radioimmunoassay kits (Diagnostic Products Corporation, Los Angeles, CA, USA).

\section{Immunohistochemical staining for PDGF}

Human placentae of both groups A and B were fixed in $4 \%$ buffered neutral formalin, dehydrated, and embedded in paraffin. Sections, 5 to $6 \mu \mathrm{m}$ in thickness, were deparaffinized followed by standard histological techniques. Immunohistochemical staining was performed by the labeled streptavidin biotin (LSAB) method (DAKO, Glostrup, Denmark) as previously described (11). Monoclonal antibody to the human PDGF-B chain at a dilution of 1:100 was used as the primary antibody.

\section{In vitro culture of vascular smooth muscle cells}

Human umbilical artery SMC were plated in a $2.1 \mathrm{~cm}^{2}$ well at an approximate density of 10000 cells/well and subcultured for $12 \mathrm{~h}$ in DMEM supplemented with 10\% FBS, penicillin G, and streptomycin sulfate. Thereafter, the cultured SMC were stepped down to steroid-free conditions by incubating in phenol red-free DMEM containing 1\% dextran-coated charcoal-stripped FBS (DCC-FBS) in the presence or absence of PDGF-BB (10 ng/ml), E2 (10-100 ng/ml), or P4 (10-500 ng/ $\mathrm{ml})$. The treatment with PDGF, E2, or P4 was started when the cultured cells were at approximately 40-50\% confluence, and monolayer cultures were maintained in phenol red-free DMEM for an additional $48 \mathrm{~h}$. At the termination of cultures, the cells were scraped off with trypsin.

\section{Immunohistochemical staining for PCNA}

To investigate the effect of PDGF on SMC proliferation and its possible interaction with sex steroid hormones, we examined the proliferative potential of SMC cultured under the above conditions by immunohistochemical staining for PCNA. Cultured SMC were fixed in 90\% ethanol. Immunohistochemical staining was performed by the LSAB method. Mouse monoclonal antibody to human PCNA at a dilution of 1:80 was used as the primary antibody. To assure the specificity of the immunological reaction, SMC in control cultures were subjected to the same LSAB method, except that the primary antibody was replaced by non-immune murine immunoglobulin G (IgG) (Miles, Erkhardt, IN, USA) at the same dilution as used with the specific antibody.
The replacement of the specific primary antibody with non-immune murine IgG resulted in a lack of positive immunostaining.

Immunostaining was analyzed in a 'blind' fashion without knowledge of the experimental group. All stained nuclei were scored as positive for PCNA. The PCNA labeling index was determined by observing more than 1000 nuclei for each experimental sample.

Data are represented as means \pm s.D. Statistical analysis was performed with Student's $t$-test. An analysis of variance was performed to assess the differences in the PCNA positive rate during the study. Scheffe's test was used to analyze the difference between means. A $P$ value $<0.05$ was considered to be significant.

\section{Results}

The serum PDGF levels in normotensive cases (group C) and severe PIH cases (group A) are shown in Fig. 1 (top panel). The serum PDGF levels in group A were significantly higher than those in group $C(P<0.01)$.

Figure $1 \mathrm{~A}$ and $1 \mathrm{~B}$ represents hematoxylin and eosin (H-E) staining and immunohistochemical staining for PDGF in the decidua from preterm delivery cases (group B). Immunohistochemical staining demonstrated that PDGF was scarcely apparent in the spiral artery in the decidua in group B. On the other hand, immunohistochemical staining in the decidua from severe $\mathrm{PIH}$ cases in group A revealed that PDGF was abundantly present in the SMC of the spiral artery in the decidua (Fig. 1D). Furthermore, the immunolocalization of PDGF in group A coincided closely with the lesion of atherosclerotic change seen with H-E staining (Fig. 1C).

Serum E2 and P4 levels in groups $\mathrm{A}$ and $\mathrm{C}$ are represented in Fig. 2. Serum E2 levels were significantly lower in group A than in group $C(P<0.01)$. There was no significant difference in serum $\mathrm{P} 4$ levels between group $\mathrm{A}$ and group $\mathrm{C}$.

Figure 3 shows the PCNA positive rate of SMC cultured in the absence or presence of PDGF, E2, or P4. Treatment with PDGF resulted in a significant increase in the PCNA positive rate in cultured SMC $(P<0.001)$ relative to that in control cultures, whereas treatment with E2 resulted in a significant decrease in the PCNA positive rate of cultured SMC $(P<0.01)$. No significant change in the PCNA positive rate was obtained with treatment with $\mathrm{P} 4$. Moreover, the increase in PCNA positive rate of cultured SMC caused by treatment with PDGF was significantly suppressed in response to concomitant treatment with PDGF and E2 $(P<0.01)$.

\section{Discussion}

Although PDGF was first purified from human platelets, it has been found to be produced by a number of other cell types, including macrophages, endothelial cells and even $\operatorname{SMC}(4,12,13)$. It is released from 

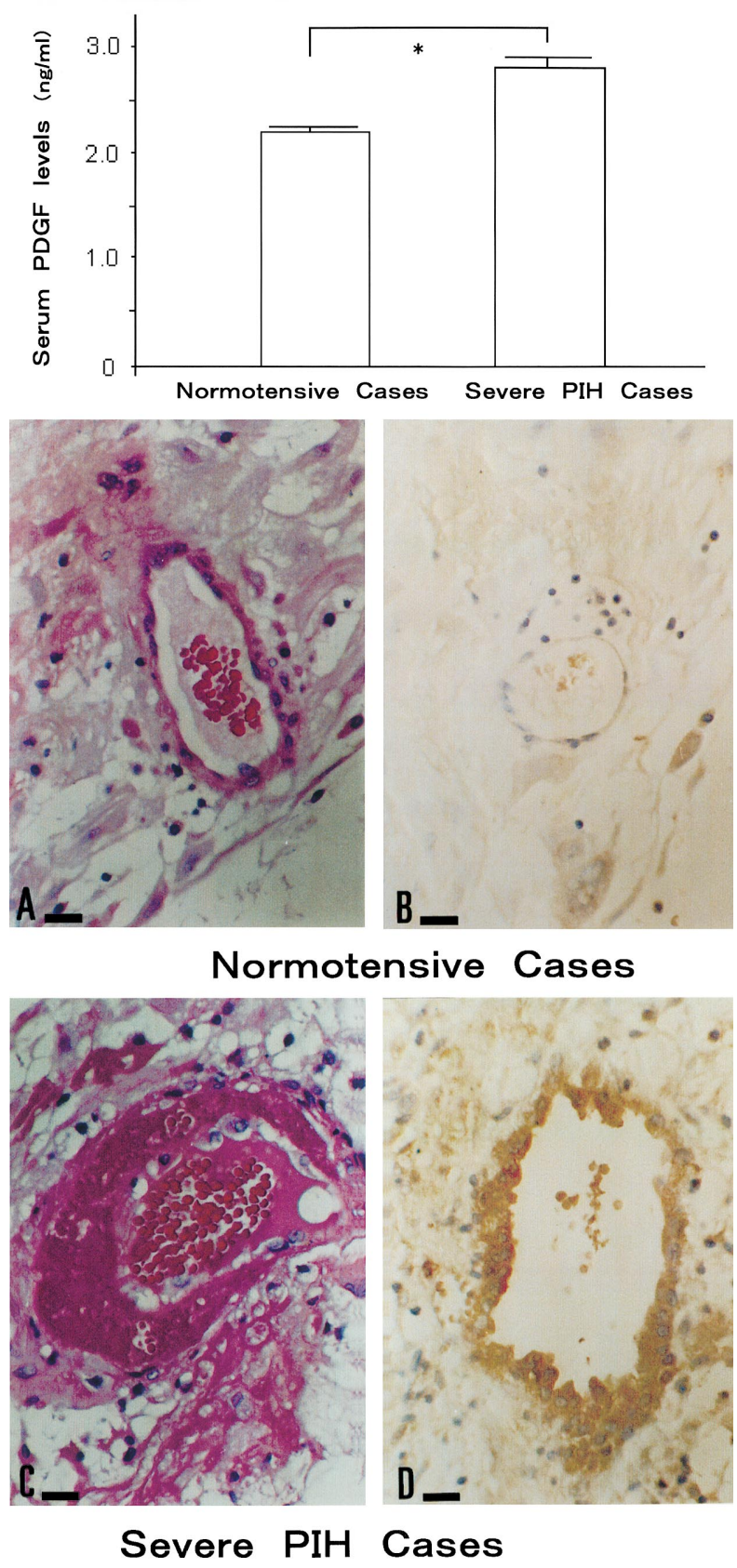

Figure 1 (Top panel) Serum PDGF levels in normotensive and severe PIH cases. ${ }^{*} P<0.01$. (Bottom panels) $\mathrm{H}$-E staining $(\mathrm{A})$ and immunohistochemical staining (B) for PDGF in the decidua of normotensive preterm delivery placenta, and $\mathrm{H}-\mathrm{E}$ staining $(\mathrm{C})$ and immunohistochemical staining (D) for PDGF in the decidua of severe $\mathrm{PIH}$ placenta. Bars $=5 \mu \mathrm{m}$. Original magnification, $\times 400$.

$\alpha$-granules of platelets when platelets aggregate, and increases proliferation of fibroblasts, SMC and glial cells in vitro. Therefore, PDGF is thought to participate in wound healing and the development of atherosclerosis $(14,15)$. Recent studies also indicate vital roles for PDGF in regulating normal and pathological cell proliferation and inflammation (16). Cultured SMC have been reported to produce PDGF and to respond to PDGF in an autocrine and paracrine manner, as a function of their developmental stage $(17,18)$.

The pathological changes of PIH in the spiral arteries at the implantation site were first described by Hertig (6). Since then, acute atherosis has become the hallmark of $\mathrm{PIH}$, and it is generally believed that the other major placental changes are the result of the altered placental bed vessels $(19,20)$. Acute atherosis in $\mathrm{PIH}$ is characterized by fibrinoid necrosis of the vessel wall, accumulation of lipid-laden macrophages, and a mononuclear perivascular infiltration (21). These changes are observed in both myometrial and decidual spiral arteries (22).

In this study, we aimed to elucidate the role of PDGF in acute atherotic lesions of the spiral artery of the decidua, characteristic of the pathophysiology of PIH. Serum PDGF levels in severe PIH cases were significantly higher than those in normotensive cases in the present study. Immunohistochemical staining demonstrated more abundant expression of PDGF in spiral arteries of the decidua in PIH cases compared with normotensive cases. These immunohistochemical localizations of PDGF closely coincided with the lesion of atherosclerotic change observed in H-E staining. Moreover, treatment with PDGF resulted in a significant increase in the PCNA positive rate of cultured SMC. These results indicate that PDGF may play a significant role in stimulating SMC proliferation in the spiral artery in PIH cases. It seems that the abundant expression of PDGF in the spiral artery may lead to higher concentrations of PDGF in serum in PIH cases compared with those in normotensive cases. Recent studies have shown greater expression of PDGF in the fetal part of the placenta in pre-eclamptic cases than in normotensive cases $(23,24)$. The present study demonstrates, for the first time, the potential role of PDGF in the decidua, which is the maternal part of the placenta, associated with PIH,

It is noteworthy that the addition of $\mathrm{E} 2$ resulted in a significant decrease in the PCNA positive rate of cultured SMC, whereas the addition of PDGF resulted in a significant increase in the PCNA positive rate of those cells. These results indicate that PDGF stimulates SMC proliferation while E2 inhibits SMC proliferation. The increase in SMC proliferation stimulated by the addition of PDGF was significantly inhibited in response to concomitant treatment with PDGF and E2. In cultured human endothelial cells, preeclamptic sera have been shown to stimulate the expression of PDGF (25). Considering the fact that serum E2 levels were lower in severe PIH cases compared with normotensive cases, our results imply that PDGF-induced proliferation of SMC may be accelerated under the endocrine milieu found in pregnant women with severe PIH.

The pathophysiology of PIH is likely to involve a variety of factors. More studies on the interaction with 

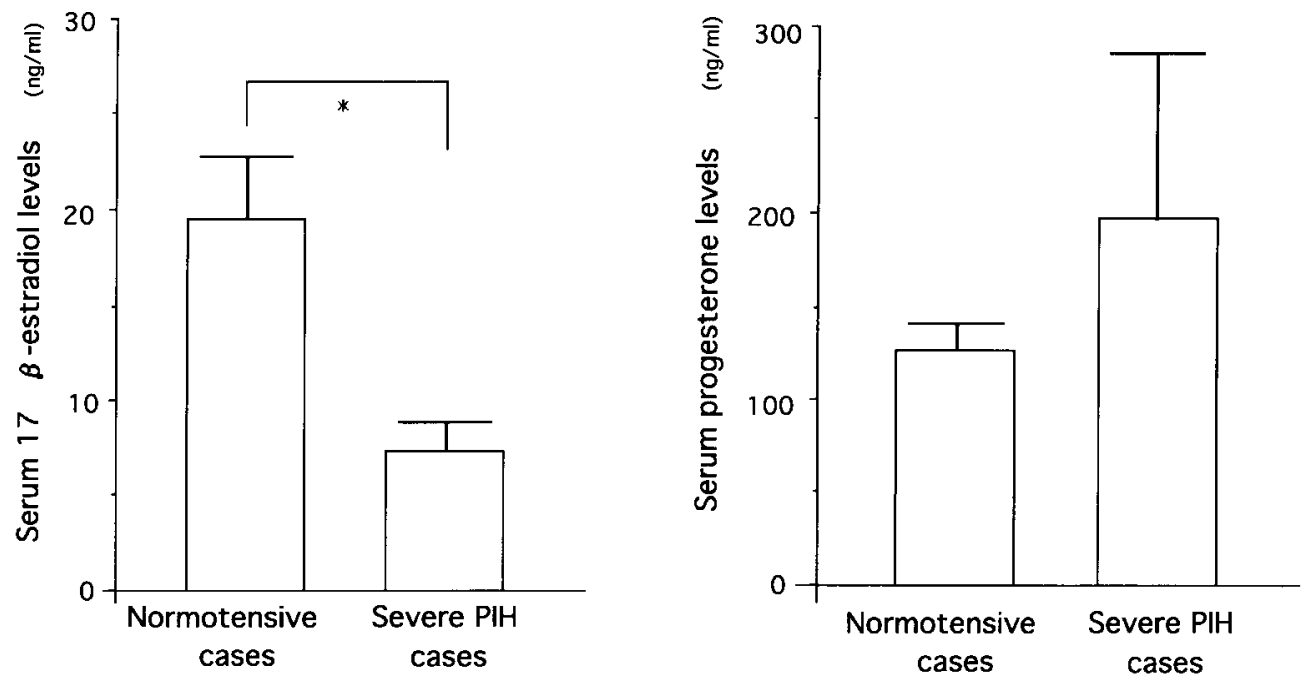

Figure 2 Serum levels of E2 (left panel) and P4 (right panel) in normotensive and severe PIH cases. ${ }^{*} P<0.01$.

other growth factors or cytokines will be needed to elucidate the molecular mechanism underlying the pathophysiology of PIH. PDGF has been found to be produced by both SMC and endothelial cells. D'Amore and Smith (26) reported that PDGF had more effect on SMC growth than on endothelial cell growth. On the basis of the abundant expression of PDGF in SMC of spiral arteries in the decidua and its stimulatory effect on vascular SMC proliferation which may elicit the atherosclerotic change in the spiral artery of the decidua, our hypothesis that PDGF may play an important role in the pathophysiology of $\mathrm{PIH}$ seems to be ensured.

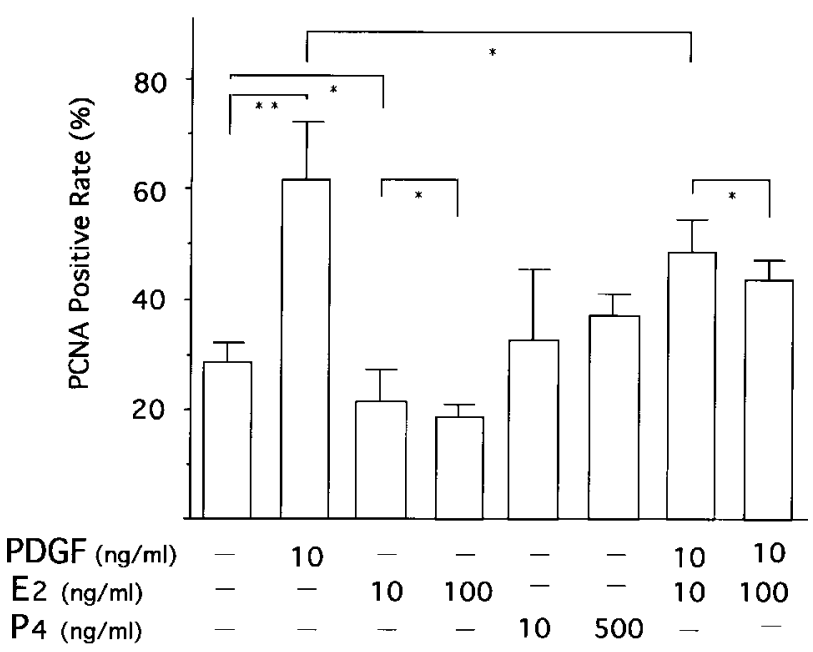

Figure 3 PCNA positive rate of human umbilical artery SMC cultured in vitro in the absence or presence of PDGF, E2, and P4. ${ }^{\star} P<0.01,{ }^{*} P<0.001$.

\section{Acknowledgements}

This work was supported, in part, by a Grant-in-Aid for Scientific Research 07457388 from the Japanese Ministry of Education, Science, and Culture and by the Japan Association of Obstetricians and Gynecologists Ogyaa-Donation Foundation (JODF).

\section{References}

1 Ross R \& Glomset JA. Arteriosclerosis and the arterial smooth muscle cell. Science $1973 \mathbf{1 8 0} 1332-1339$.

2 Goustin AS, Betsholtz C, Pfeifer-Ohlsson S, Persson H, Rydnert J, Bywater $\mathrm{M}$ et al. Coexpression of the sis and myc proto-oncogenes in developing human placenta suggests autocrine control of trophoblast growth. Cell 198541 301-312.

3 Deuel TF. Polypeptide growth factors: role in normal and abnormal cell growth. Annual Review of Cell Biology 19873 443-492.

4 Ross R, Raines EW \& Bowen-Pope DF. The biology of plateletderived growth factor. Cell 198646 155-169.

5 Zeek PM \& Assali NS. Vascular changes in the decidua associated with eclamptogenic toxemia of pregnancy. American Journal of Clinical Pathology 195020 1099-1109.

6 Hertig AT. Vascular pathology in hypertensive albuminuric toxemias of pregnancy. Clinics $1945 \mathbf{4} 602-614$.

7 The American College of Obstetricians and Gynecologists. Hypertension in pregnancy. In ACOG Technical Bulletin, vol 219, pp 1-8, Washington DC: American College of Obstetricians and Gynecologists, 1996.

8 Berthois Y, Katzenellenbogen JA \& Katzenellenbogen BS. Phenol red in tissue culture media is a weak estrogen: implications concerning the study of estrogen responsive cells in culture. PNAS $1986832496-2500$.

9 Welshons WV, Wolf MF, Murphy CS \& Jordan VC. Estrogenic activity of phenol red. Molecular and Cellular Endocrinology 1988 $\mathbf{5 7} 169-178$.

10 Shiraishi T, Morimoto S, Itoh K, Sato H, Sugihara K, Onishi T et al. Radioimmunoassay of human platelet-derived growth factor using monoclonal antibody towards a synthetic 73-97 fragment of its B-chain. Clinica Chimica Acta 1989184 115-174.

11 Elias JM, Margiotta M \& Gaborc D. Sensitivity and detection efficiency of the peroxidase antiperoxidaze (PAP), avidin-biotin 
peroxidase complex (ABC), peroxidase-labeled avidin-biotin (LSB) methods. American Journal of Clinical Pathology 198992 62-67.

12 Heldin CH, Wasteson A \& Westermark B. Platelet-derived growth factor. Molecular Cell Biology 198539 169-187.

13 Shimokado K, Raines EW, Madtes DK, Barrett TB, Benditt EP \& Ross R. A significant part of macrophage-derived growth factor consists of at least two forms of PDGF. Cell $1985 \mathbf{4 3}$ 277-286.

14 Ross R. The pathogenesis of atherosclerosis: a perspective for the 1990s. Nature $1993362801-809$.

15 Ross R \& Glomset JA. The pathogenesis of atherosclerosis. New England Journal of Medicine 1976295 369-377, 420-425.

16 Deuel TF. Structural and functional diversity of the plateletderived growth factor. Current Opinions in Biotechnology 19912 802-806.

17 Golden MA, Au YP, Kirkman TR, Wilcox JN, Raines EW, Ross R et al. Platelet-derived growth factor activity and mRNA expression in healing vascular grafts in baboons. Journal of Clinical Investigation $1991 \mathbf{8 7} 406-414$.

18 Majesky MW, Reidy MA, Bowen-Pope DF, Hart CE, Wilcox JN \& Schwartz SM. PDGF ligand and receptor gene expression during repair of arterial injury. Journal of Cell Biology $19901112149-$ 2158.

19 Robertson WB, Brosen I \& Dixon G. Uteroplacental vascular pathology. European Journal of Obstetrics, Gynecology and Reproductive Biology 19755 47-65.

20 Benirschke K \& Kaufmann P. Hypertensive disorders. In Pathology of the Human Placenta, edn 2, ch 22, pp 499-530. Eds K
Benirschke \& P Kaufmann. Berlin, Heidelberg, New York: Springer-Verlag, 1990.

21 De Wolf F, Robertson WB \& Brosens I. The ultrastructure of acute atherosis in hypertensive pregnancy. American Journal of Obstetrics and Gynecology $1975 \mathbf{1 2 3}$ 164-174.

22 Meekins JW, Pijnenborg R, Hanssens M, McFadyen IR \& van Assche A. A study of placental bed spiral arteries and trophoblast invasion in normal and severe pre-eclamptic pregnancies. British Journal of Obstetrics and Gynaecology $1994101669-674$.

23 Gurski MR, Gonzalez E \& Brown EG. Immunochemical localization of platelet-derived growth factor in placenta and its possible role in pre-eclampsia. Journal of Investigative Medicine 199947 128-133.

24 Jurcovicova J, Krueger KS, Nandy I, Lewis DF, Brooks GG \& Brown EG. Expression of platelet-derived growth factor-A mRNA in human placenta: effect of magnesium infusion in preeclampsia. Placenta 199819 423-427.

25 Taylor RN, Musci TJ, Rodgers GM \& Roberts JM. Preeclamptic sera stimulate increased platelet-derived growth factor mRNA and protein expression by cultured human endothelial cells. American Journal of Reproductive Immunology 199125 105-108.

26 D'Amore PA \& Smith SR. Growth factor effects on cells of the vascular wall: a survey. Growth Factors 19938 61-75.

Received 16 June 2000

Accepted 19 October 2000 